\title{
Innovative Therapie, frühe Nutzenbewertung und Wirtschaftlichkeit
}

\author{
Christian Dierks
}

Dierks + Bohle, Rechtsanwälte, Berlin, Deutschland

Am 1. Januar 2011 ist das Gesetz zur Neuordnung des Arzneimittelmarktes (AMNOG) in Kraft getreten. Ziel des AMNOG ist es unter anderem, die steigenden Arzneimittelausgaben in der gesetzlichen Krankenversicherung einzudämmen.

Der Preis innovativer Arzneimittel wird seit Inkrafttreten des AMNOG nur noch in den maximal ersten 12 Monaten nach Markteinführung durch den pharmazeutischen Unternehmer frei festgesetzt. Im Anschluss an die frühe Nutzenbewertung wird entweder ein Festbetrag festgesetzt oder der Preis auf Basis einer Vereinbarung zwischen dem jeweiligen pharmazeutischen Unternehmer und dem Spitzenverband Bund der Krankenkassen in Abhängigkeit vom Zusatznutzen für die Patienten im Verhältnis zur «zweckmäßigen Vergleichstherapie» festgelegt. Der Zusatznutzen eines Arzneimittels im Sinne des AMNOG ist der patientenrelevante therapeutische Effekt, insbesondere hinsichtlich der Verbesserung des Gesundheitszustands, der Verkürzung der Krankheitsdauer, der Verlängerung des Überlebens, der Verringerung von Nebenwirkungen oder einer Verbesserung der Lebensqualität, der quantitativ oder qualitativ höher ist als der Nutzen der zweckmäßigen Vergleichstherapie. Die Nutzenbewertung führt der Gemeinsame Bundesausschuss (GBA) auf der Grundlage eines vom pharmazeutischen Unternehmer eingereichten Dossiers durch, in dem dieser, vorrangig durch Ergebnisse aus randomisierten klinischen Prüfungen, den Zusatznutzen nachzuweisen hat. Der GBA kann das Institut für Qualität und Wirtschaftlichkeit im Gesundheitswesen (IQWiG) oder Dritte mit der Nutzenbewertung beauftragen. Über Vorliegen und (gegebenenfalls) Umfang des Zusatznutzens entscheidet final aber der GBA durch Beschluss, der sodann Teil der Arzneimittelrichtlinien wird.

Wird ein Zusatznutzen festgestellt und kommt innerhalb von 6 Monaten nach Beschlussfassung des GBA über Vorliegen und gegebenenfalls Umfang des Zusatznutzens keine Einigung über den Erstattungsbetrag zustande, setzt eine eigens hierfür eingerichtete Schiedsstelle den Erstattungsbetrag fest. Dieser Erstattungsbetrag gilt über einen gesetzlichen Verweis auch im Bereich der privaten Krankenversicherung (PKV) und Beihilfe. Bei nicht belegtem Zusatznutzen wird das Arzneimittel einer Festbetragsgruppe zugeordnet.

Die Erstattungspreisvereinbarung zwischen dem pharmazeutischen Unternehmer und dem Spitzenverband Bund der Krankenkassen soll nach den gesetzlichen Vorgaben vorsehen, dass Verordnungen des betroffenen Arzneimittels im Rahmen von Wirtschaftlichkeitsprüfungen als Praxisbesonderheiten von der Prüfungsstelle anerkannt werden, soweit der Arzt bei der Verordnung im Einzelfall die dafür vereinbarten Anforderungen an die Verordnung eingehalten hat. Nach Abschluss einer solchen Erstattungspreisvereinbarung können Arzneimittel im Hinblick auf eine Wirtschaftlichkeitsprüfung also privilegiert sein. Vor Abschluss einer entsprechenden Erstattungspreisvereinbarung folgt die Wirtschaftlichkeitsprüfung demgegenüber den allgemeinen Regeln, und Praxisbesonderheiten werden nur unter den «regulären» Bedingungen anerkannt, was nach der höchstrichterlichen Rechtsprechung voraussetzt, dass ein spezifischer, vom Durchschnitt der Vergleichsgruppe signifikant abweichender Behandlungsbedarf der Patientenklientel (nicht einzelner kostenintensiver Krankheitsfälle) und die hierdurch hervorgerufenen Mehrkosten nachgewiesen werden.

Soweit nach Abschluss des Frühnutzenbewertungsverfahrens ein Erstattungspreis für ein Arzneimittel vereinbart oder durch die Schiedsstelle festgesetzt wird und dieser gegebenenfalls unterhalb des bislang durch das pharmazeutische Unternehmen in den ersten 12 Monaten frei festgesetzten Preises für das Arzneimittel liegt, hat dies nicht zur Folge, dass die zurückliegenden Verordnungen des Arzneimittels in den vergangenen 12 Monaten automatisch in der Höhe der Preisdifferenz zwischen frei festgesetztem und nunmehr vereinbartem oder durch die Schiedsstelle festgesetztem Erstattungsbetrag als unwirtschaftlich anzusehen wären.

\section{Disclosure Statement}

Der Autor war Referent beim «2. Interdisziplinären Expertenforum Hautkrebs», das von Bristol-Myers Squibb veranstaltet und unterstützt wurde.

\begin{tabular}{ll}
\hline KARGER & $\begin{array}{l}\text { (1) 2012 S. Karger GmbH, Freiburg } \\
0378-584 \mathrm{X} / 12 / 3514-0007 \$ 38.00 / 0\end{array}$ \\
$\begin{array}{l}\text { Fax }+497614520714 \\
\text { Information@Karger.de } \\
\text { www.karger.com }\end{array}$ & $\begin{array}{l}\text { Accessible online at: } \\
\text { www.karger.com/onk }\end{array}$
\end{tabular}

